\title{
Pattern and prevalence of neonatal thrombocytopenia in Port Harcourt, Nigeria
}

\section{Zaccheaus A Jeremiah' Justina E Oburu²}

'Hematology and Blood Transfusion Science Unit, Department of Medical Laboratory Sciences, College of Health Sciences, Niger Delta University, Wilberforce Island, Bayelsa State, Nigeria; ${ }^{2}$ Department of Hematology and Blood Transfusion University of Port Harcourt Teaching Hospital, Port Harcourt, Nigeria
Correspondence: Zaccheaus Awortu Jeremiah

P.O. Box 1437, Diobu, Port Harcourt, 50000 I, Nigeria

Tel +2348034045636

Email zacjerry@yahoo.com
This article was published in the following Dove Press journal:

Pathology and Laboratory Medicine International

16 April 2010

Number of times this article has been viewed

Background: In Port Harcourt, evidence-based guidelines for platelet transfusion therapy in neonatal patients are yet to be defined and the prevalence and pattern of neonatal thrombocytopenia has not yet been reported.

Methods: Platelet counts of 132 neonatal patients admitted into the special care baby unit (SCBU) at the University of Port Harcourt Teaching Hospital in Nigeria were assessed using the International Committee on Standards in Hematology (ICSH) approved manual procedures for hemocytometry. ${ }^{6}$

Study design: This is a cross sectional study carried out on neonates to determine the prevalence and pattern of neonatal thrombocytopenia.

Results: The median platelet count of the neonates was $97.0 \times 10^{9} / \mathrm{L}$ (interquartile range [IQR] 50-152) while the mean age was 61.7 hours (range 1-336 hours). The overall prevalence of neonatal thrombocytopenia was 53.0\%. Mild thrombocytopenia (platelet count 51-100 $\times 10^{9} / \mathrm{L}$ ) was found in $39.4 \%$ of the neonates, $12.1 \%$ had moderate thrombocytopenia (platelet count $30-50 \times 10^{9} / \mathrm{L}$ ), while severe thrombocytopenia (platelet count $<30 \times 10^{9} / \mathrm{L}$ ) was detected in $1.5 \%$ of the neonates. Of these, $84.84 \%$ of the cases occurred within 72 hours (early onset). The most common clinical diagnosis among the neonates was severe birth asphyxia (33.3\%), followed by neonatal jaundice (19.7\%), neonatal sepsis (16.7\%), low birth weight (13.6\%), anemia and bleeding $(6.1 \%)$, and other clinical conditions (10.6\%). There was no association between clinical diagnosis and thrombocytopenia (Fisher's exact test $=10.643 ; P=0.923$ ).

Conclusion: There is a high prevalence of early onset neonatal thrombocytopenia cases in this region of Nigeria, the majority of which are mild in nature. There is a need to define a safer lower limit for platelet count and to determine which neonates will benefit from treatment.

Keywords: neonatal thrombocytopenia, platelet count, thrombocytopenia, Port Harcourt, Nigeria

\section{Introduction}

Thrombocytopenia is defined as platelet count less than $150 \times 10^{9} / \mathrm{L}$ and sometimes indicated as less than $100 \times 10^{9} / \mathrm{L} .{ }^{1}$ However, it is considered severe if the platelet count is below $30 \times 10^{9} / \mathrm{L}$, moderate if it is between $30 \times 10^{9} / \mathrm{L}$ and $50 \times 10^{9} / \mathrm{L}$ and mild (usually asymptomatic) if above $50 \times 10^{9} / \mathrm{L} .^{2}$ The finding of thrombocytopenia is reported to be common among neonates and often causal. In the majority of cases, it is found following bleeding and could also be detected following thromboembolic events that can occur in the antiphospholipid syndrome and sometimes in heparin-induced thrombocytopenia. ${ }^{3,4}$

There are many causes of neonatal thrombocytopenia ranging from sepsis, neonatal jaundice, and alloantibody. One of the causes of severe thrombocytopenia 
known to affect neonates is the acquired thrombocytopenia caused by alloantibodies such as neonatal alloimmune thrombocytopenia. ${ }^{1}$ Fetal and neonatal alloimmune thrombocytopenia is due to an incompatibility of platelet antigens between parents and the transplacental passage of maternal alloantibodies against a variety of platelet antigens, most frequently anti-HPA-1a present on the platelets of the fetus and father, but absent in the mother. This occurs in approximately 1 in every 1000-5000 live births. ${ }^{5}$

In this setting, the prevalence of neonatal thrombocytopenia is not yet reported and platelet transfusion is not yet in active practice. There is paucity of data on the prevalence and causes of thrombocytopenia in neonates in this environment. This study was undertaken to investigate the prevalence of thrombocytopenia and to ascertain if there is any relationship between clinical presentation and thrombocytopenia in neonates.

\section{Materials and methods Subjects}

A total of 132 neonatal patients admitted into the special care baby unit (SCBU) with various clinical diagnoses at the University of Port Harcourt Teaching Hospital constituted the study population. Fifty-six of the neonates were males while 76 were females. Subjects were recruited consecutively in the SCBU ward within the months of July-October, 2009. The consultant pediatrician assisted in providing the clinical provisional diagnosis and in obtaining the consent of the parents. There was a trained hospital phlebotomist attached to the ward who assisted in sample collection. The study received institutional ethical approval from the University of Port Harcourt Teaching Hospital.

\section{Procedures}

Two milliliters of venous blood was collected from each neonate into an ethylenediaminetetracetic acid (EDTA) anticoagulant and kept on a roller mixer for constant mixing until a platelet count was performed using the International Committee on Standards in Hematology (ICSH) approved procedures. ${ }^{6}$ Whole blood $(20 \mu \mathrm{L})$ was diluted with $0.38 \mathrm{~mL}$ of $1 \%$ ammonium oxalate reagent. Platelets were counted under the microscope using the improved Neubaeur counting chamber. Results were expressed as platelet $\times 10^{9} / \mathrm{L}$. As a quality control measure, a thin smear of each sample was made and stained with Giemsa stain. The Giemsa-stained film was examined under the light microscope to rule out platelet clumps and to ensure that the platelets were spread evenly before the actual count was done by a trained biomedical scientist/hematologist. The 132 samples reported in this study were those that were free of platelet clumps, hence any error which may have been due to falsely low platelet count was eliminated.

\section{Statistics}

Descriptive statistics of continuous variables were expressed in mean, median, and interquartile range (IQR) using the Statistical Package for Social Sciences (version 15; SPSS Inc, Chicago, IL). Fisher's exact test was used to test the association between the provisional clinical diagnoses with thrombocytopenia. $P$ values less than or equal to 0.05 were considered statistically significant.

\section{Results}

One hundred thirty-two newborns of both sexes (56 males [42.4\%] and 76 females [57.6\%]) admitted into the SCBU of the University of Port Harcourt Teaching Hospital (UPTH), Port Harcourt, Nigeria with various provisional diagnoses had their platelet count done in the Hematology Department of the same hospital as part of the medical process of investigation and treatment. The most common provisional diagnosis was severe birth asphyxia (33.3\%), followed by neonatal jaundice (19.7\%), neonatal sepsis (16.7\%), low birth weight (13.6\%), anemia and bleeding (6.1\%). Other clinical conditions such as multiple congenital anomalies, cephalohematoma, obstructive adenoid, and mecomium aspiration accounted for the remaining $10.6 \%$ (Table 1 ). The mean age of the neonates was 61.7 hours (range $1-336$ hours) while the median (IQR) platelet count was $97.0 \times 10^{9} / \mathrm{L}(50-152)$ (Table 2 ).

Fisher's exact test analysis did not reveal any association between the provisional diagnosis and thrombocytopenia. (Fisher's exact test $=10.643 ; P=0.923$ ) as shown in Table 3 . There was a high prevalence $(84.84 \%)$ of early onset

Table I Gender characteristics and provisional clinical diagnosis of the neonates

\begin{tabular}{lll}
\hline Characteristics & Number & Percentage \\
\hline Gender & 56 & \\
Male & 76 & 42.4 \\
Female & & 57.6 \\
Clinical diagnosis & 8 & \\
Anemia/bleeding & 18 & 6.1 \\
Low birth weight & 26 & 13.6 \\
Neonatal jaundice & 22 & 19.7 \\
Neonatal sepsis & 44 & 16.7 \\
Severe birth asphyxia & 14 & 33.3 \\
Others & & 10.6 \\
\hline
\end{tabular}


Table 2 Mean age and median platelet counts of the neonates

\begin{tabular}{lll}
\hline Statistics & Platelet count $\times 10^{9} / \mathrm{L}$ & Age (hours) \\
\hline Mean (SD) & $112.91(65.6)$ & 61.7 \\
Median (IQR) & $97.0(50-152)$ & - \\
Minimum & 24 & 1 \\
Maximum & 312 & 336 \\
\hline
\end{tabular}

Abbreviations: SD, standard deviation; IQR, interquartile range.

( $<72 \mathrm{hrs}$ ) of neonatal thrombocytopenia as shown in Table 4. Severe thrombocytopenia (platelet count $<30 \times 10^{9} / \mathrm{L}$ ) was detected in $1.5 \%$ of the neonates. Moderate thrombocytopenia (platelet count $30-50 \times 10^{9} / \mathrm{L}$ ) accounted for $12.1 \%$. Mild thrombocytopenia (platelet count $51-100 \times 10^{9} / \mathrm{L}$ ) was detected in $39.4 \%$. The remaining $47.0 \%$ had normal platelet counts of above $100 \times 10^{9} / \mathrm{L}$. Overall, the prevalence of thrombocytopenia (mild, moderate, and severe) in this neonatal population was $53.0 \%$ (Figure 1). Neonatal platelet serology was not done to rule out platelet alloimmunization due to lack of a facility for testing.

\section{Discussion}

Physiologically, a healthy fetus has a platelet count of greater than $150 \times 10^{9} / \mathrm{L}$ by the second trimester of pregnancy and only $2 \%$ of term infants are thrombocytopenic at birth. Severe thrombocytopenia (platelet count $<50 \times 10^{9} / \mathrm{L}$ ) has been reported to occur in fewer than three per 1000 term infants and most of the causes has been linked to alloimmune thrombocytopenia. ${ }^{7}$ Recently, the same group of authors ${ }^{7}$ has reported that thrombocytopenia is one of the most common hematological problems in neonates, affecting at least $25 \%$ of all admissions to neonatal intensive care units (NICU). ${ }^{8}$ Thrombocytopenia occurs in up to a third of preterm neonates admitted to intensive care units and two patterns of presentation have been identified: early onset, occurring within 72 hours of birth, and late onset thrombocytopenia, which develops after 72 hours. ${ }^{9}$

In the present study, the prevalence of neonatal thrombocytopenia was $53.0 \%$, of which $39.4 \%$ were mild, $12.1 \%$ moderate and $1.5 \%$ severe and the mean age of the neonates was 61.7 hours. The prevalence of neonatal thrombocytopenia in this locality therefore seems high when compared with studies from other places. ${ }^{10,11}$ The major mechanism underlying neonatal thrombocytopenia, accounting for about $75 \%$ of cases, has been attributed to impaired platelet production while increased platelet consumption and/or sequestration accounts for the remainder of cases. ${ }^{12}$ Numerous conditions are associated with low platelet counts. Newborns who suffer hypoxia or acidosis after birth trauma often develop thrombocytopenia. This is due to hypoxic injury caused to fetal megakaryocytes which cause the progenitor cell to be driven toward the erythroid series at the expense of leucocytes and platelets. ${ }^{11}$ Newborns of mothers with maternal hypertension are also prone to early onset thrombocytopenia due to disorders associated with placental insufficiency. ${ }^{9,11}$ In this study, severe birth asphyxia was seen as the most common provisional diagnosis (33.3\%) among these neonates.

The second most common diagnosis among the neonates in this study was neonatal jaundice $(19.7 \%)$, followed by neonatal sepsis $(16.7 \%)$. More than $80 \%$ of neonates with proven infections become thrombocytopenic. These infections may be bacterial, fungal, or viral. ${ }^{10}$ Bacterial infection causes endothelial damage, thus accelerating destruction of platelets and their removal by the reticuloendothelial system. At the time bacterial infection is diagnosed, $25 \%$ of neonates will already have low platelet counts. By $36-48$ hours later, virtually all will be thrombocytopenic. ${ }^{13}$ The average duration of bacterial infection-mediated thrombocytopenia has been reported to be 6 days. $^{13}$

Table 3 Association of provisional diagnosis with thrombocytopenia

\begin{tabular}{|c|c|c|c|c|}
\hline \multirow[t]{2}{*}{ Diagnosis } & \multicolumn{4}{|c|}{ Thrombocytopenia (× 109/L) } \\
\hline & Severe $(<30)$ n (\%) & Moderate (30-50) n (\%) & Mild (5 I-100) n (\%) & Normal (>100) n (\%) \\
\hline Anemia/bleeding & $0(0)$ & $0(0)$ & $4(50.0)$ & $4(50.0)$ \\
\hline LBW & $0(0)$ & $2(11.1)$ & $8(44.4)$ & $8(44.4)$ \\
\hline NNJ & $0(0)$ & $0(0)$ & $12(46.2)$ & $14(53.8)$ \\
\hline NNS & $2(9.1)$ & $4(18.2)$ & $8(36.4)$ & $8(36.4)$ \\
\hline SBA & $0(0)$ & $8(18.2)$ & $14(31.8)$ & $22(50.0)$ \\
\hline Others & $0(0)$ & $2(14.3)$ & $6(42.9)$ & $6(42.9)$ \\
\hline Total & $2(1.5)$ & $16(12.1)$ & $52(39.4)$ & $62(47.0)$ \\
\hline \multicolumn{5}{|c|}{ Fisher's Exact test $=10.643, P=0.923^{\text {ns }}$} \\
\hline
\end{tabular}

Abbreviations: LBW, low birth weight; NNJ, neonatal jaundice; NNS, neonatal sepsis; SBA, severe birth asphyxia; ns, not significant. Note: Percentages were calculated within diagnosis. 
Table 4 Onset of NT among the neonates

\begin{tabular}{lll}
\hline Pattern of NT & Number (\%) & Most frequent causes \\
\hline Early onset ( $<72$ hours) & I I $(84.84)$ & SBA (33.3\%) \\
& & NNS (29.6\%) \\
Late onset ( $>72$ hours) & $20(15.16)$ & NNJ (60.0)
\end{tabular}

Abbreviations: NT, neonatal thrombocytopenia; SBA, severe birth asphyxia; NNS, neonatal sepsis; NNJ, neonatal jaundice.

Bacterial sepsis causes thrombocytopenia by other mechanisms which include disseminated intravascular coagulation (DIC), immune-mediated destruction and platelet aggregation due to bacterial products adhering to platelet membrane, and decreased platelet production from infected bone marrow. ${ }^{10}$ Viral infections in the perinatal period cause severe thrombocytopenia as a result of sialic acid loss from platelet membranes due to viral neuraminidase, intravascular platelet aggregation, and decreased production from degeneration of megakaryocytes. ${ }^{10,11}$ Late onset thrombocytopenia is often severe and prolonged, requiring treatment with platelet transfusion. ${ }^{9,14}$ Other provisional diagnoses encountered in this study population were low birth weight (13.6\%), and anemia/bleeding (6.1\%), while clinical conditions such as chromosomal abnormality, which may contribute to neonatal thrombocytopenia, accounted for $4 \% .{ }^{15}$ Statistically, no association was established between the clinical diagnosis and thrombocytopenia, so it is difficult to conclude that the high prevalence rate of thrombocytopenia in this study is due to these clinical conditions. However, neonatal sepsis may contribute largely to the cause of most severe thrombocytopenia in this region.

Neonatal thrombocytopenia could also be as a result maternal idiopathic thrombocytopenia (ITP). Maternal antibodies in this disorder are directed against "public" antigen on the platelets (usually glycoprotein IIb/IIIa) and antibody-coated platelets are cleared by the reticuloendothelial system, causing maternal thrombocytopenia. The fetus is affected because maternal antibodies cross the placenta

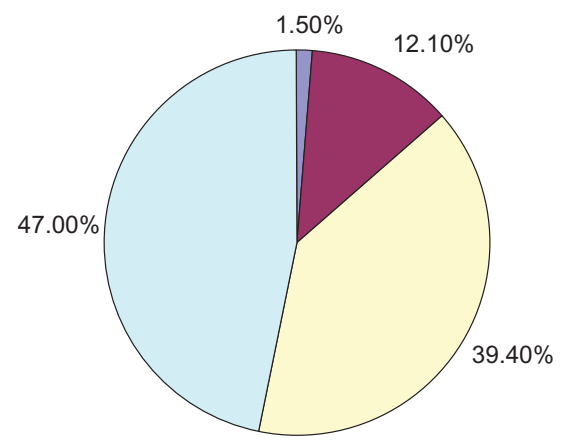

$\square$ Severe

$\square$ Moderate

$\square$ Mild

$\square$ Normal

Figure I Prevalence and severity of neonatal thrombocytopenia among neonates in Port Harcourt, Nigeria. and bind to the same public antigens on fetal platelets. ${ }^{11}$ Another condition to consider is neonatal alloimmune thrombocytopenia (NAIT), which is the platelet equivalent of hemolytic disease of the newborn due to red blood cell $\mathrm{RhD}$ incompatibility. This occurs when a mother lacks a platelet antigen that her fetus has inherited from the father. As a result, maternal immunoglobulin $\mathrm{G}$ antibodies, formed against the foreign antigen on fetal platelets, cross the placenta and destroy fetal platelets. ${ }^{10,15}$ Both maternal ITP and NAIT can result in severe thrombocytopenia and affected fetuses and neonates are always at high risk for serious bleeding complications. ${ }^{10}$

The most commonly identified antibody in sensitized Caucasian women is anti-HPA-1a which accounts for $80 \%$ to $90 \%$ of NAIT cases. ${ }^{10,15-17}$ In Port Harcourt, Nigeria, there are no facilities for platelet typing and antibody identification so the alloimmune status of these neonates is not known. Statistical analysis by Fisher's exact test to relate the provisional diagnosis to the prevalence of neonatal thrombocytopenia did not reveal any association between the two. It is possible the majority of the cases of neonatal thrombocytopenia may be of immune origin, which was not detected. This underscores the need to initiate platelet antibody screening among neonates in special baby care units as a means to define thrombocytopenia due to immune causes. In this study, we noted that the two cases $(1.5 \%)$ of severe thrombocytopenia were in neonates who had neonatal sepsis. It could also be deduced that most cases of severe thrombocytopenia (platelet count $<30 \times 10^{9} / \mathrm{L}$ ) in this locality may be due to neonatal sepsis. Early onset of neonatal thrombocytopenia is an indication of placental insufficiency and reduced platelet production. ${ }^{12}$

Laboratory evaluation is vital to providing clues to the kinetic mechanism of the infants' thrombocytopenia. Introduction of mean platelet volume (MPV), which is the measure of the average size of circulating platelets, could be useful to assess when thrombocytopenia is caused by reduced production or accelerated destruction of platelets. ${ }^{17}$ In reduced production, the MPV is normal (7.5-9.5 fL) while in accelerated destruction, the MPV is elevated $(>10-12 \mathrm{fL})$. Larger platelets are evident when the bone marrow is stimulated to produce more immature platelets in response to increase platelet utilization.

The percentage of reticulated platelets (RPs) is another indicator of the platelet kinetic mechanism. RPs are newly produced platelets that have a higher RNA content than do older platelets. ${ }^{13} \mathrm{RP} \%$ is low $(<2 \%)$ when platelet production is low, and high $(>10 \%)$ when platelets are being 
consumed at an accelerated rate. Another useful parameter is the measurement of plasma thrombopoietin (Tpo) growth factor. Tpo is the primary regulator of platelet production in the in the neonate. Its level in the plasma is useful in differentiating thrombocytopenia caused by low platelet production and accelerated platelet destruction. Tpo which is made in the liver is removed from the blood by binding to Tpo receptors on megakaryocytic progenitors, megakaryocytes, and platelets. When platelet production is abnormally low, fewer megakaryocytes are produced and plasma Tpo is high. Although, Tpo quantity is typically used as a research tool, its measurement may prove very useful in detecting neonatal thrombocytopenia in the future. ${ }^{10,17}$

\section{Acknowledgments}

We wish to acknowledge the cooperation of the nurses in the neonatal SCBU unit, the consultant pediatrician, Dr Lucy Y Ide and the laboratory staff of the Hematology Department. Without their efforts, this study would not have been possible. Mr Jude Asolo helped in analyzing the data.

\section{Disclosures}

The authors declare that they have no competing interests, financial or otherwise, with this work.

\section{References}

1. Veneri D, Franchini M, Randon F, Nichele I, Pizzolo G, Ambrosetti A. Thrombocytopenia: a clinical point of view. Blood Transfus. 2009;7: $75-85$.

2. Ruggeri M, Fortuna S, Rodeghiero F. Heterogeneity of terminology and clinical definitions in adult idiopathic thrombocytopenic purpura: a clinical appraisal from a systematic review of literature. Hematologica. 2008;93:98-103.
3. Diz-Kuciikaya R, Hacihanefioglu A, Yenerel M, et al. Anti-phospholipid antibodies and antiphospholipid syndrome in patients presenting with immune thrombocytopenic purpura: a prospective cohort study. Blood. 2001;98:1760-1764.

4. Warkentin TE. An overview of the heparin-induced thrombocytopenia syndrome. Semin Thromb Hemost. 2004;30:273-283.

5. Dreyfus M, Kaplan C, Verdy E, et al. Frequency of immune thrombocytopenia in newborns: a prospective study. Immune Thrombocytopenia Working Group. Blood. 1997;89:4402-4406.

6. International Committee for Standardization in Hematology (ICSH) handbook WB Saunders: Philadelphia; 1996. p. 7.

7. Roberts IA, Murray NA. Neonatal thrombocytopenia: new insights into pathogenesis and implications for clinical management. Curr Opin Pediatr. 2001;13:16-21.

8. Roberts I, Stanworth S, Murray NA. Thrombocytopenia in the neonate. Blood Rev. 2008;22:173-186.

9. Chakravorty S, Murray N, Roberts I. Neonatal thrombocytopenia. Early Hum Dev. 2005;81:35-41.

10. Jones CW. Platelet Disorders, NBIN. 2004;4:181-190.

11. Wong W, Glader B. Approach to the newborn who has thrombocytopenia. Neoreviews. 2004;5:e444-e450.

12. Roberts I, Murray NA. Neonatal thrombocytopenia: causes and management. Arch Dis Child Fetal Neonatal Ed. 2003;88:F359-F364.

13. Sola MC. Evaluation and treatment of severe and prolonged thrombocytopenia in neonates. Clin Perinatol. 2004;31:1-14.

14. Murray NA, Howarth LJ, McCloy MP, Letsky EA, Roberts IA. Platelet transfusion in the management of severe thrombocytopenia in neonatal intensive care units patients. Transfus Med. 2002;12:35-41.

15. Stanworth SJ, Clarke P, Watts T, et al. Prospective, observational study of outcomes in neonates with severe thrombocytopenia. Pediatrics. 2009; 124:e826-e834.

16. Rehman A. Immune thrombocytopenia in children with reference to low-income countries. East Mediterr Health J. 2009;15:729-737.

17. Christensen R. Evaluation and treatment of thrombocytopenia in the NICU. Program and Abstracts of the National Association of Neonatal Nurses. 22nd Annual Conference, 2006 Nov 8-11; Nashville, TN, USA.
Pathology and Laboratory Medicine International

\section{Publish your work in this journal}

Pathology and Laboratory Medicine International is a peer-reviewed, open access journal focusing on innovative basic research and translational research related to pathology or human disease. The journal includes original research, updates, case reports, reviews and commentaries on current controversies. The Academic Sponsor

\section{Dovepress}

of this journal is the Chinese American Pathology Association (CAPA). The manuscript management system is completely online and includes a very quick and fair peer-review system. Visit http://www.dovepress.com/testimonials.php to read real quotes from published authors. 